\title{
Performance analysis in cognitive radio system under perfect spectrum sensing
}

\author{
Chen Song, Gu Shuainan, Zhang Yankui
}

Zhengzhou Institute of Information Science and Technology, Zhengzhou 450001, China

\begin{abstract}
Keywords: cognitive radio; spectrum sensing ; continuous time markov chain, transmit collision; channel utilization
\end{abstract}

\begin{abstract}
Sensing period and optimization of user access in cognitive radio system are analyzed. By introducing birth and death process into cognitive radio system, a model of the queuing of primary users is built, based on which the probability of spectrum sensing and how the re-enter of the primary users effect on the spectrum usage of cognitive radio users are studied theoretically. Simulation results have justified the fitness of the model of joint analysis in the practical environment. Therefore we may conclude that it will achieve the highest overall spectrum efficiency by setting up configurations in a cognitive system according to the theory above.
\end{abstract}

\section{Introduction}

According to the fact that the spectrum is in short and used efficiently, cognitive radio technologies gets the free spectrum resources and uses them by sensing technology under the premise that Primary User (PU) with spectrum authorization has no sense ${ }^{[1]}{ }^{[2]}$. In order to improve the efficiency of cognitive radio technology resources, according to the business needs, user access characteristics, spectrum sensing methods and some other characteristics of the cognitive system, system modeling, parameter optimization, performance analysis and some other researches were made in the existing literatures.

In [3], the appearing characteristic of PU was described by Continuous-Time Markov Chain (CTMC). Cumulative time and distribution probability of PU's occupying channel were met by analyzing. In [4] and [5], cognitive radio system was system modeled by state5's and state8's CTMC. Then, Secondary User (SU) access optimization algorithm was put forward and the spectrum utilization efficiency and user fairness were in good compromise. In [6], according to the SU's coexistence of different types, the system was modeled by Markov model stack. Markov chains of different states were established and were connected by few core states. The method simplified the modeling and analysis of SU of multiple types' cognitive radio systems. In [7], cognitive radio system model was established based on restaurant game theory and Semi-Markov process. The analysis complexity of cognitive radio system was reduced effectively under the subjective effect and system analysis frame was constructed based on social dynamic influence. In [8], for VolP business, general Markov chain model was established and adjacent transition relations of states are solved. User connection properties and data packet loss performance were analyzed. The simulation shows the system model was effective.

Markov chain was introduced to model for the cognitive radio system in the literals above. System performance has been analyzed and optimized for parameters and conditions of different systems. It can be seen that the continuous-time Markov chain can describe the cognitive radio system very well. Based on this characteristic, under ideal testing conditions, this paper has conducted the system model with CTMC-N. System general equation is established and the system of equations of general solution is given based on "flow equilibrium" theory. Finally, relations between collision probability, channel utilization, SU arrival identity the number of channels and the false detection probability are analyzed and described through the numerical simulation of the system.

\section{System modeling}

The running state and running process of the system have been described and part of system 
performance was analyzed by continuous time Markov chain model in [9]. Make the assumption that $i$ is the amount of PU's occupied channel, $j$ is the amount of PU's occupied channel, the amount of system is $M$ and $i+j \leq M, i \geq 0, j \geq 0$. According to the definition of continuous Markov chain, set state $(i, j)$ as the general condition, then the state transition relations of the model is as shown in Figure 1.

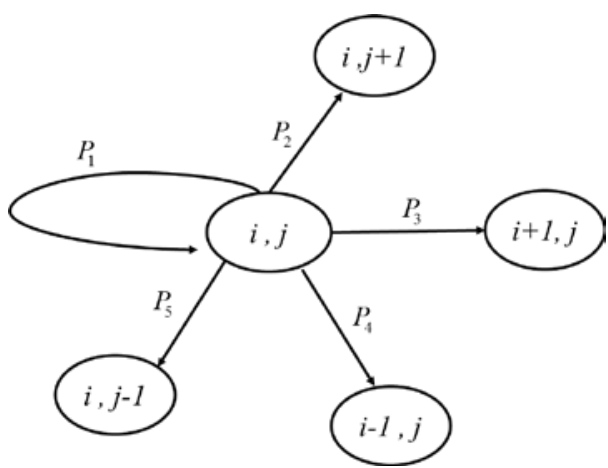

Figure 1 System state transition diagram

Assume that the spectrum detection of SU on PU is ideal testing. There are $M$ authorized channels in PU system and channels are distributed and controlled by control center. PU and SU systems are independent of each other. When SU detects that the authorized channel of PU is free, it selects a random channel for data transmission. We assume that arrival probability of PU and SU obeys Poisson distribution with parameters $\lambda_{p}$ and $\lambda_{s}$, and the service time also obeys the exponential distribution with parameter $\mu_{p}$ and $\mu_{s}$. The number of PU and SU is much larger than the number of authorized channels.

\section{Modeling and performance analysis}

\section{Solve transition probability}

According to the system model of Figure 1, in order to obtain the distribution probability of states, the transition probability needs to be solved. Transition probabilities were solved as follows,

1. Get $P_{1}$

$P_{1}$ represents that the amount of PU and SU is unchangeable and the system state is transmitting at the same place. There may be e possible reasons.

(1) When the new PU arrives, the total $M$ channels were occupied by PU, that is $i=M$ The probability is:

$$
P_{1-1}=P(M, 0)
$$

(2) When the new PU arrives, a channel which SU is using is occupied. The probability is:

$$
P_{1-2}=\frac{j}{M-i}
$$

(3) When the new PU arrives, the total $M$ channels were occupied by PU where $i$ channels were occupied by PU and $j$ channels were occupied by PU. And $i+j=M$. The probability is:

$$
P_{1-3}=P(i, j) \quad i+j=M
$$

So, $P_{1}$ can be expressed as:

$$
P_{1}=\lambda_{p}\left(P_{1-1}+P_{1-2}\right)+\lambda_{s} P_{1-3}
$$

In the above 3 situations, PU and SU have collisions in (2), which will result in the loss of system performance.

2. get $P_{2}$

$P_{2}$ represents that the new arrival SU occupied a free channel successfully. The probability is:

$$
P_{2}=\lambda_{s}
$$

3. get $P_{3}$

$P_{3}$ represents that the new arrival PU occupied a free channel successfully and doesn't cause 
interference to other users in the system. The transition probability is:

$$
P_{3}=\lambda_{p} \frac{M-i-j}{M-i}
$$

4. get $P_{4}$

$P_{4}$ represents that PU was served and left. The transition probability is:

$$
P_{4}=i \mu_{p}
$$

5. get $P_{5}$

An SU was served and left. The transition probability is:

$$
P_{5-1}=j \mu_{s}
$$

6. Solve states' stationary distribution

Having obtained the transition probability, linear equations of CTMC is $\mathbf{P Q}=0$, where $\mathbf{P}=\left\{P_{i, j} \mid i \geq 0, j \geq 0, i+j \leq M\right\}$ which represents the probability vector of states' stationary distribution. $\mathbf{Q}$ represents the transition probability vector. $\mathbf{P}$ can be expressed :

$$
\mathbf{P}=\left(P_{0,0}, \cdots P_{0, j}, \cdots P_{0, M}, P_{1,0}, \cdots, P_{1, M-1}, P_{2,0}, \cdots \cdots, P_{i, j}, \cdots \cdots, P_{M, 0}\right)
$$

The vector $\mathbf{Q}$ can be expressed:

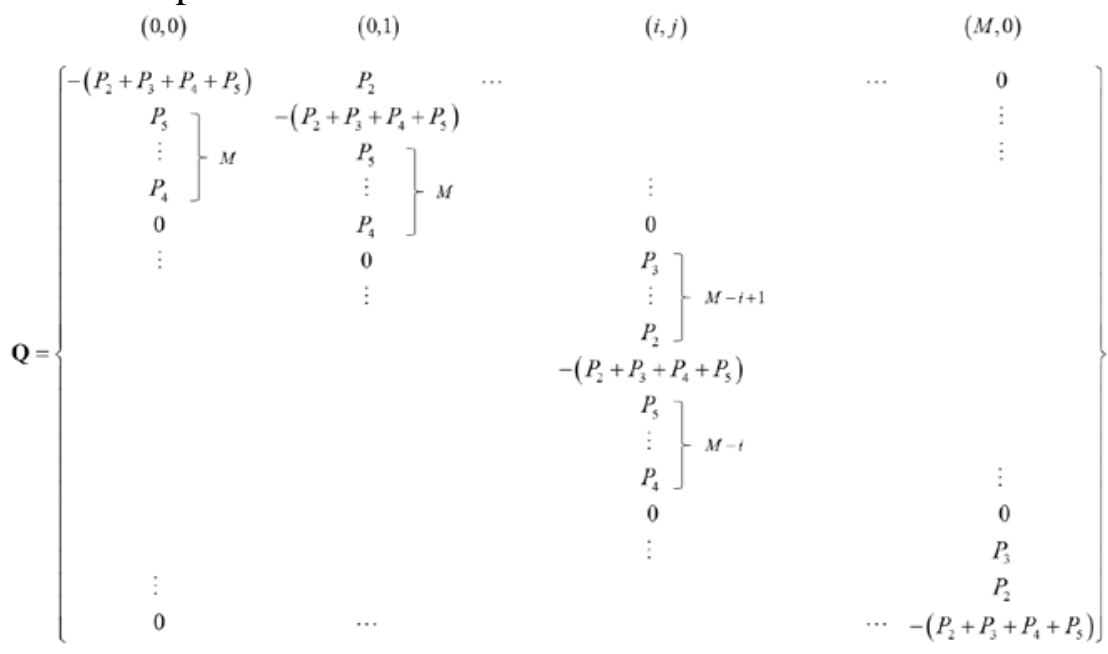

The elements of $\mathbf{P}$ sums up 1, which can be expressed:

$$
\sum_{i} \sum_{j} P_{i, j}=1
$$

Equations can be solved by replacing the elements of last line of $\mathbf{Q}$ with 1 , then the new vector $\mathbf{Q}^{*}$ can be obtained, that is:

$$
\mathbf{P Q}^{*}=\mathbf{b}
$$

Where $\mathbf{b}=(0,0, \cdots \cdots, 1), \mathbf{Q}$ is a square matrix of $(M+1)(M+2) / 2$, so $\mathbf{P}$ can be solved by matrix inversion.

\section{System collision probability and spectrum utilization}

$$
\mathbf{P}=\mathbf{b Q}^{*-1}
$$

In the cognitive radio system, the system spectrum utilization and users' collision probability are the important evaluation indexes for system performance. The increase of spectrum utilization is the original intention of cognitive radio system. The collision probability of users' can not only influence system' spectrum utilization, but also influence the need of different users' businesses. Assuming that SU is ideally perceived, the main SU collision seizes the channel SU is using only when PU arrives. Then the collision probability is:

$$
P_{\text {collision }}=\sum_{i, j} \frac{P_{i, j} P_{1-2} \lambda_{p}}{P_{1}+P_{2}+P_{3}+P_{4}+P_{5}}
$$

The definition of SU system spectrum utilization is:

$$
\Lambda=\frac{\lambda_{s}}{\mu_{s}}\left(1-P_{\text {collision }}\right)
$$


Where, $\frac{\lambda_{s}}{\mu_{s}}$ is the traffic intensity of SU.

\section{Performance simulation and analysis}

The numerical simulation analysis was based on the system model above in this part. The simulation conditions are set as follows: PU service business strength was based on VoIP (Voice over Internet Protocol ${ }^{[10]}$. That is $P_{H_{0}}=0.65, P_{H_{1}}=\lambda_{p} / \mu_{p}=0.35, \mu_{p}=1, \mu_{s}=2$ 。

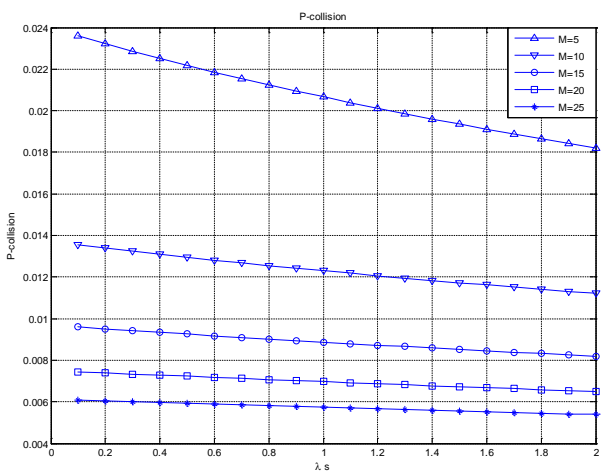

Figure 2 collision probability under different number of channels

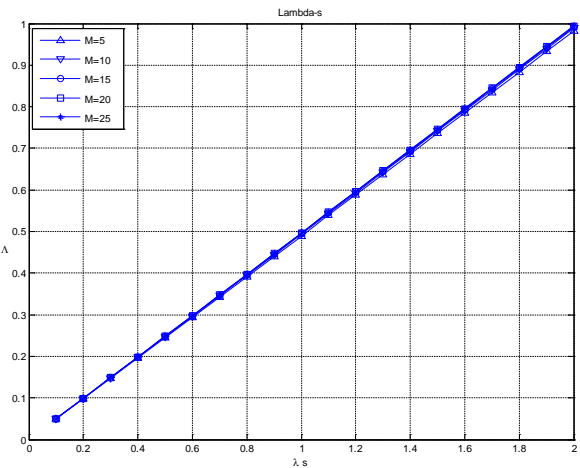

Figure 3 collision probability under different number of channels

Figure 2 and Figure 3 show that under different numbers of channels, the arrival intensity variation has influence on system collision probability and spectrum utilization. Figure 2 and Figure 3 show that with the increase of channel, the collision probability of users decreases obviously. This is because the arrival intensity of PU is certain. With the number of channels increases, the probability of free channel increases. Under the ideal sensing conditions, collision probability between users is not high and has few influences on overall channel utilization.

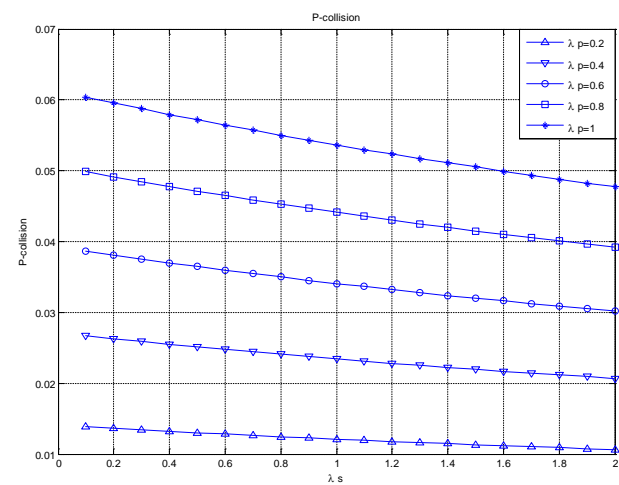

Figure 4 collision probability under different PU access rate 


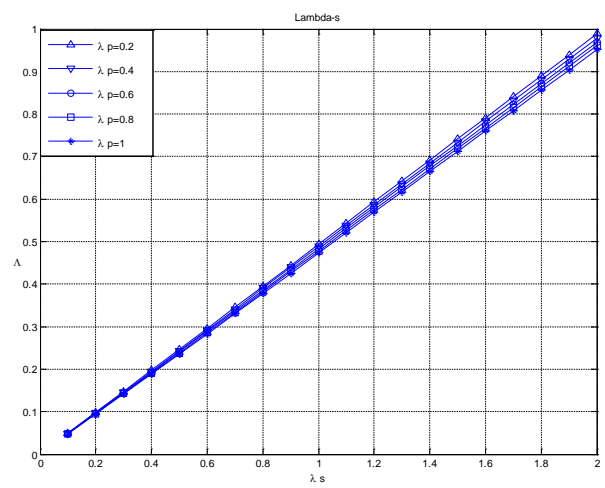

Figure 5 collision probability under different PU access rate

Figure 4 and Figure 5 show that under different arrival intensity of PU, the arrival intensity variation of SU has influence on system collision probability and spectrum utilization. Figure 4 and Figure 5 show that with arrival intensity of PU increases, channel utilization decreases and the collision probability gradually increases. This is because the appearing probability of PU is higher; the probability of seizing SU channel is higher, which caused more loss on channel utilization.

From the simulation results above, under the ideal spectrum sensing conditions, channel utilization increases with the arrival intensity of SU increases while system collision probability increases with the arrival identity of SU increases. However, collision between users causes little loss on system spectrum utilization.

\section{Conclusion}

This paper models and analyzes on cognitive radio system under ideal sensing conditions based on continue time markov model, the relations between number of system channels, arrival intensity of PU and SU, the channel utilization and main SU collision probability were obtained by numerical simulation. When design cognitive radio system according with the system model above, the model and conclusion can provide system parameter setting and performance optimization compromise with some reference and direction. However, in the paper, only modeling under ideal sensing conditions was taken into account. So the following main task is system modeling and analysis under non-ideal sensing conditions.

\section{Reference}

[1] Federal Communications Commission. Notice of proposed rule making and order: Facilitating opportunities for flexible, efficient, and reliable spectrum use employing cognitive radio technologies [J]. ET docket, 2005 (03-108): 73.

[2] Haykin S. Cognitive radio: brain-empowered wireless communications [J]. Selected Areas in Communications, IEEE Journal on, 2005, 23(2): 201-220.

[3] Lu L, Zhou X, Li G Y. Optimal sequential detection in cognitive radio networks[C]//Wireless Communications and Networking Conference (WCNC), 2012 IEEE. IEEE, 2012: 289-293.

[4] Wang B, Ji Z, Liu K J R. Primary-prioritized Markov approach for dynamic spectrum access[C]//New Frontiers in Dynamic Spectrum Access Networks, 2007. DySPAN 2007. 2nd IEEE International Symposium on. IEEE, 2007: 507-515.

[5] Wu Y, Wang B, Liu K J R, et al. Repeated open spectrum sharing game with cheat-proof strategies[J]. Wireless Communications, IEEE Transactions on, 2009, 8(4): 1922-1933.

[6] Li X, Xiong C. Markov model bank for heterogenous cognitive radio networks with multiple dissimilar users and channels[C]//Computing, Networking and Communications (ICNC), 2014 International Conference on. IEEE, 2014: 93-97.

[7] Jiang C, Chen Y, Yang Y, et al. Dynamic Chinese Restaurant Game: Theory and Application to Cognitive Radio Networks[J]. Wireless Communications, IEEE Transactions on, 2014, 13(4): 1960-1973. 
[8] Castellanos-Lopez S, Cruz-Perez F, Rivero-Angeles M, et al. Joint Connection Level and Packet Level Analysis of Cognitive Radio Networks with VoIP Traffic[J]. Selected areas in communications , IEEE Journal on , 2014,32(3):601-614.

[9] Chu T, Phan H, Zepernick H. Dynamic Spectrum Access for Cognitive Radio Networks with Prioritized Traffics[J]. Communications Letters, IEEE ;120114,18(7): 1218

[10]Pei Y, Hoang A T, Liang Y C. Sensing-throughput tradeoff in cognitive radio networks: how frequently should spectrum sensing be carried out? [C]//Personal, Indoor and Mobile Radio Communications, 2007. PIMRC 2007. IEEE 18th International Symposium on. IEEE, 2007: 1-5. 